\title{
Analytical sensitivity matrix for the inverse identification of hardening parameters of metal sheets
}

\author{
P.A. Prates*, A.F.G. Pereira, M.C. Oliveira, J.V. Fernandes \\ CEMMPRE - Department of Mechanical Engineering, University of Coimbra, Rua Luís Reis Santos, Pinhal de Marrocos, 3030-788, Coimbra, Portugal
}

\section{A R T I C L E I N F O}

\section{Keywords:}

Analytical sensitivity matrix

Gradient-based optimization methods

Inverse methods

Parameter identification

Hardening laws

Finite-element analysis

\begin{abstract}
A B S T R A C T
An analytical approach is proposed for calculating the sensitivity matrix in inverse gradient-based optimization problems, enabling the identification of the isotropic and kinematic hardening laws parameters of metal sheets. The approach was tested for inverse identification problems involving the circular bulge test, the biaxial tensile test on a cruciform specimen and the reverse shear test, and compared with forward finite differences. The proposed strategy proved to be accurate and an expeditious alternative to the use of finite differences for computing the Jacobian matrix, in case the objective function is defined using global variables such as pressure and force.
\end{abstract}

\section{Introduction}

Finite Element Analysis (FEA) is now a well-established computational tool in the automotive and aeronautics industries for the optimization of metal forming processes. The manufacture of components with increasingly complex geometries and tight tolerances demands an increase in accuracy of numerical simulation results. In this sense, the accurate description of the material behaviour depends not only on the flexibility of the constitutive model selected for the material, but also on the strategy adopted to identify its parameters. Identification strategies are generally seen as optimization problems, where the purpose is to minimize the difference between computed and experimental measurements of one or more experiments. Among the inverse strategies, the most common is the Finite Element Model Updating (FEMU) (Avril et al., 2008; Prates et al., 2016a). FEMU consists on performing successive finite element simulations of the mechanical test (or tests) in order to minimize the difference between the experimental and the numerical measurements. This difference is expressed by an objective function and its minimization requires efficient and robust optimization algorithms, which iteratively act on the values of the material parameters. Gradient-based optimization algorithms like the Gauss-Newton and the Levenberg-Marquardt algorithms (Marquardt, 1963) are the most widely used within inverse identification strategies, as they generally do not require a very large number of evaluations of the objective function (Bäker and Shrot, 2013). However, each iteration step involves the calculation of the gradient of the objective function with respect to the material parameters (i.e. the Jacobian matrix), which represents the sensitivity of the computed results from experiments to the parameters to be identified. Typically, the Jacobian matrix is numerically approximated by finite differences and, at each iteration, its evaluation requires at least as many numerical simulations as the number of parameters to be identified. Adjoint-based methods are also available for computing the Jacobian matrix (Cooreman, 2008); however, these methods are not as widely used as finite differences and require implementation in the numerical simulation code, which is not always possible in case of commercial codes.

Some authors proposed alternative methods for computing the sensitivities, within the context of inverse identification strategies, mainly to overcome the potentially high computational costs associated with the use of finite differences. Endelt and Nielsen (2004), Endelt et al. (2005), Endelt and Danckert (2009), Cooreman (2008) and Cooreman et al. (2007) explored the possibility of analytically calculating the sensitivity matrix for the inverse identification of the yield criterion and hardening law parameters. Endelt and co-authors proposed a general approach for the inverse identification of material parameters based on global measurements (i.e. punch force) of circular or square cup forming processes (Endelt and Nielsen, 2004, Endelt et al., 2005; Endelt and Danckert, 2009). The use of a gradient-based algorithm, together with the analytical definition of the sensitivity matrix, allowed a fast and stable identification of the material parameters, with a reduced number of finite element simulations. However, the large amount of numerical simulation data required for the analytical calculation of the sensitivity matrix makes this type of approach efficiently usable only when integrated within the code used in the

\footnotetext{
* Corresponding author.

E-mail address: pedro.prates@dem.uc.pt (P.A. Prates).
} 
numerical simulation; in addition, it is not always possible to implement subroutines in commercial numerical simulation codes. Moreover, the data writing and saving can be time and resource consuming and thus affect the approach efficiency, particularly for numerical models with a high number of elements. Nevertheless, this type of analytical formulation is simple, since it avoids considering local variables such as strain fields. Cooreman (2008) applied a finite element based inverse method to identify the material parameters describing the plastic behaviour of metal sheets, based on mechanical tests of samples with complex geometries and/or loading conditions. The objective function expresses the difference between experimental and numerical strain fields, for several load points, and it is minimized using gradient-based algorithms. The analytically calculated Jacobian matrix resorts to local stress and strain data available from the FE simulation. The author concluded that, in case of homogeneous deformation, the sensitivities obtained by means of the proposed analytical approach were in agreement with those obtained through finite differentiation. Otherwise, in case of complex, heterogeneous deformation, it was no longer possible to accurately compute the sensitivities, requiring too many iterations to be competitive with finite differentiation. Bäker and Shrot (2013) proposed an alternative method for solving inverse identification problems using auxiliary quantities, which overcomes the requirement of performing numerical simulations to compute the sensitivity matrix. For this purpose, the experimental and numerical variables used in the objective function should be proportional to the auxiliary quantities that are analytically evaluated from the parameters to be identified. The calculation of the auxiliary quantities is timeconsuming, particularly when a high number of finite elements are used. Also, the proper choice of the auxiliary quantities can be complex and requires the proper physical knowledge of the mechanical problem.

This work presents an analytical approach to calculate the sensitivity matrix, for solving inverse problems concerning the identification of the hardening parameters of metal sheets. This approach resorts to a small amount of numerical simulation data and allows the stability and convergence of the optimization procedure. The optimization appeals to objective functions that express the difference between experimental and numerical global results (e.g. load or pressure $v s$. displacement). The analytical approach is able to compute the sensitivity of the global variables of the mechanical test, even though heterogeneous deformation occurs in the specimen. Additionally, the implementation of the analytical approach aims to be efficient and user friendly, avoiding the use of large amounts of data and the need to implement subroutines, even when a high number of finite elements is used. The analytical approach is stablished using numerical simulations of the circular bulge test. The sensitivity of pressure $v s$. pole height results to variations of the hardening law parameters is evaluated, which allows supporting the procedure for analytically calculating the sensitivity matrix. The performance of the proposed Jacobian approximation scheme is numerically tested and its results are compared with those obtained via finite differences, under three different inverse identification problems involving the use of the following tests: (i) circular bulge test (Reis et al., 2017a); (ii) biaxial tensile test on a cruciform specimen (Prates et al., 2014, 2016b) and (iii) reverse shear test (Pereira et al., 2015). The proposed analytical approach proves to be expeditious in relation to the finite differences method, within its scope of application that will be duly defined.

\section{Framework}

Inverse parameter identification strategies are generally seen as optimization problems, where the difference between computed and reference results of one or more experiments is minimized. This difference is expressed by an objective function, $f(\mathbf{A})$, and its minimization is commonly performed using gradient-based optimization algorithms, which iteratively updates the vector of constitutive parameters to optimize, A. Objective functions are commonly formulated under the concept of least squares, as follows:

$f(\mathbf{A})=\frac{1}{m} \sum_{i=1}^{m}\left(r_{i}(\mathbf{A})\right)^{2}$

where $m$ is the total number of measuring instants and $r_{i}(\mathbf{A})$ is the residual at the instant $i$. The residuals can be expressed in terms of relative differences, $r_{i}(\mathbf{A})=\left(g_{i}{ }^{N u m}(\mathbf{A})-g_{i}^{R e f}\right) / g_{i}^{R e f}$ or absolute differences, $r_{i}(\mathbf{A})=g_{i}{ }^{N u m}(\mathbf{A})-g_{i}^{R e f}$, where $g_{i}^{N u m}(\mathbf{A})$ and $g_{i}^{\text {Ref }}$ are the numerically predicted and the reference result for the instant $i$. In this work, $g_{i}{ }^{\text {Num }}(\mathbf{A})$ and $g_{i}{ }^{\text {Ref }}$ are global variables measured at the instant $i$.

The minimization of $f(\mathbf{A})$ resorts to the gradient-based LevenbergMarquardt algorithm (Marquardt, 1963), expressed as follows:

$\mathbf{A}^{s+1}=\mathbf{A}^{s}-\left(\mathbf{J}^{\mathrm{T}} \mathbf{J}+\lambda \operatorname{diag}\left(\mathbf{J}^{\mathrm{T}} \mathbf{J}\right)\right)^{-1} \mathbf{J}^{\mathrm{T}} \mathbf{r}\left(\mathbf{A}^{s}\right)$,

where $s$ is the iteration step, $\mathbf{J}$ is the Jacobian matrix, $\mathbf{r}\left(\mathbf{A}^{s}\right)$ is the vector of residuals and $\lambda$ is the damping factor, which is updated after each iteration according to the convergence rate (see Marquardt (1963)). Each term of the Jacobian matrix, , is defined as:

$J_{i j}=\partial r_{i}\left(A_{j}\right) / \partial A_{j}$,

with $i=1, \ldots, m$ and $j=1, \ldots, p$, where $m$ is the total number of measuring instants and $p$ is the total number of constitutive parameters; $A_{j}$ is the constitutive parameters, with index $j$, of the vector of constitutive parameters, A. In case the residuals are expressed in terms of absolute or relative differences, each term of the Jacobian matrix can be computed via forward finite differences, respectively, as follows:

$J_{i j}=\frac{g_{i}^{N u m}\left(A_{j}+\delta A_{j}\right)-g_{i}^{N u m}\left(A_{j}\right)}{\delta A_{j}}$

$J_{i j}=\frac{g_{i}^{N u m}\left(A_{j}+\delta A_{j}\right)-g_{i}^{N u m}\left(A_{j}\right)}{g_{i}^{N u m}\left(A_{j}\right) \delta A_{j}}$,

where $\delta A_{j}$ is a small perturbation value on the constitutive parameter, $A_{j}$. The full computation of the Jacobian via finite differences requires as many numerical simulations of $g_{i}{ }^{N u m}\left(A_{j}+\delta A_{j}\right)$ as the number of constitutive parameters to be optimized.

In this work, the Levenberg-Marquardt algorithm is used to optimize the hardening parameters of the elastoplastic constitutive model, assuming: (i) the elastic behaviour described by the generalised Hooke's law; (ii) the plastic behaviour described by Hill'48 yield criterion (Hill, 1948), and isotropic and kinematic hardening described by Swift (1952) and Lemaitre-Chaboche (1989) laws, respectively. The Hill'48 yield surface is described by:

$$
\begin{aligned}
& F\left(\Sigma_{\mathrm{yy}}-\Sigma_{\mathrm{zz}}\right)^{2}+G\left(\Sigma_{\mathrm{zz}}-\Sigma_{\mathrm{xx}}\right)^{2}+H\left(\Sigma_{\mathrm{xx}}-\Sigma_{\mathrm{yy}}\right)^{2}+2 L \Sigma_{\mathrm{yz}}{ }^{2}+2 M \Sigma_{\mathrm{xz}}{ }^{2} \\
& \quad+2 N \Sigma_{\mathrm{xy}}{ }^{2}=Y^{2},
\end{aligned}
$$

where $F, G, H, L, M$ and $N$ are the material parameters describing the anisotropy of the metal sheet; $\Sigma_{\mathrm{xx}}, \Sigma_{\mathrm{yy}}, \Sigma_{\mathrm{zz}}, \Sigma_{\mathrm{xy}}, \Sigma_{\mathrm{xz}}$ and $\Sigma_{\mathrm{yz}}$ are the components of the effective stress tensor, $\Sigma$, defined in the principal axes of the orthotropy system and equal to $\sigma^{\prime}-\mathbf{X}^{\prime}\left(\sigma^{\prime}\right.$ and $\mathbf{X}^{\prime}$ are the deviatoric Cauchy stress tensor and the deviatoric back-stress tensor, respectively); $Y$ represents the flow stress and its evolution during deformation, $Y=Y(\bar{\varepsilon})$, is described by the Swift hardening law, written in the following form:

$Y=C\left(\left(Y_{0} / C\right)^{1 / n}+\bar{\varepsilon}\right)^{n}$,

where $\bar{\varepsilon}$ is the equivalent plastic strain and $C, Y_{0}$ and $n$ are the material parameters describing the hardening behaviour of the metal sheet, where $Y_{0}$ is the yield stress. The translational velocity of the yield surface centre, $\dot{\mathbf{X}}^{\prime}$, is defined by the Lemaitre-Chaboche kinematic hardening model expressed by:

$\dot{\mathbf{X}}^{\prime}=C_{X}\left(X_{\text {Sat }} \frac{\sigma^{\prime}-\mathbf{X}^{\prime}}{\bar{\sigma}}-\mathbf{X}^{\prime}\right) \dot{\bar{\varepsilon}}, \quad \mathbf{X}^{\prime}(0)=0$, 
where $\dot{\bar{\varepsilon}}$ is the equivalent plastic strain rate, $\bar{\sigma}$ is the equivalent stress and $C_{X}$ and $X_{S a t}$ are the kinematic hardening parameters representing, respectively, the rate of saturation and the saturation value of the exponential kinematic hardening, which is given by (Prates et al., 2015):

$X=X_{\text {Sat }}\left(1-\exp \left(-C_{X} \bar{\varepsilon}\right)\right)$.

\section{Analytical approach of the Jacobian matrix: an example}

The development of the analytical approach for calculating the Jacobian matrix is supported by numerical simulation results of the hydraulic bulge test with circular dies. This mechanical test allows achieving relatively high values of plastic strain before necking, enabling the proposed approach to be tested up to large plastic strain values.

The numerical model of the circular bulge test was previously developed and optimized (Reis et al., 2017b). The geometry of the tools considered in the test is schematically shown in Fig. 1, where $R_{M}=75 \mathrm{~mm}$ is the die radius, $R_{1}=13 \mathrm{~mm}$ is the die profile radius, $R_{D}=95 \mathrm{~mm}$ is the radius of the central part of the drawbead and $R_{S}=100 \mathrm{~mm}$ is the radius of the circular sheet. This geometry was built based on the experimental bulge test used by Santos et al. (2010).

The tools were described using Bézier surfaces, considering only one quarter of the geometry due to material and geometrical symmetry conditions. Additionally, the drawbead geometry was neglected and its effect was replaced by a boundary condition imposing radial displacement restrictions on nodes placed at a distance equal to $R_{D}$ from the centre of the circular sheet. The contact with friction between the sheet and the blank holder and the die was described by the Coulomb law with a constant friction coefficient of 0.02 (Reis et al., 2017a, 2017b, 2017c). The blank sheet discretization was previously optimized, describing the central region of the specimen with a regular and uniform grid discretization in the sheet plane using quadrangular elements, as represented in Fig. 2 (Reis et al., 2017b). A total of 10584 3D solid 8 node elements with two layers of elements through thickness were used (see Fig. 2 (b)); the initial sheet thickness is $1.0 \mathrm{~mm}$. All numerical simulations were carried out with the in-house code DD3IMP ${ }^{1}$ (Menezes and Teodosiu, 2000; Oliveira et al., 2008), assuming an incremental increase of the pressure applied to the sheet inner surface.

\subsection{Sensitivity analysis}

The sensitivity of the circular bulge test results to variations of the Swift hardening law parameters is studied based on numerical simulations of a fictitious reference material, whose parameters are those identified for a DP600 dual-phase steel sheet, by Teodosiu and Bouvier (2001). Three additional materials that differ from the reference material by the individual increase of $0.5 \%$ in $Y_{0}, C$ and $n$, are also used and hereafter designated as " $\delta Y_{0}$ ", " $\delta C$ " and " $\delta n$ ", respectively (see Table 1). This increment is within the range of typical values used to compute the Jacobian matrix via finite differences (Cooreman, 2008). In all materials of this table, the Hill' 48 yield parameters were kept fixed and equal to those of the reference material. The elastic properties are: Young's modulus, $E=210 \mathrm{GPa}$ and Poisson's ratio, $\nu=0.30$.

The sensitivity analysis compares, at the same pole height value, the numerical results of pressure and flow stress at the pole for the reference material with those obtained from the materials designated by " $\delta Y_{0}$ ", " $\delta C$ " and " $\delta n$ ", in terms of relative differences. Fig. 3 (a) shows the evolutions of these relative differences with the pole height. According to these results, increasing separately $0.5 \%$ in each parameter $Y_{0}, C$ and $n$, similarly affects both variables, pressure and flow stress at the pole. This can be expressed by the following equality between the relative difference in pressure and flow stress:

\footnotetext{
${ }^{1}$ DD3IMP: Contraction of Deep Drawing 3D IMPlicit finite element code.
}

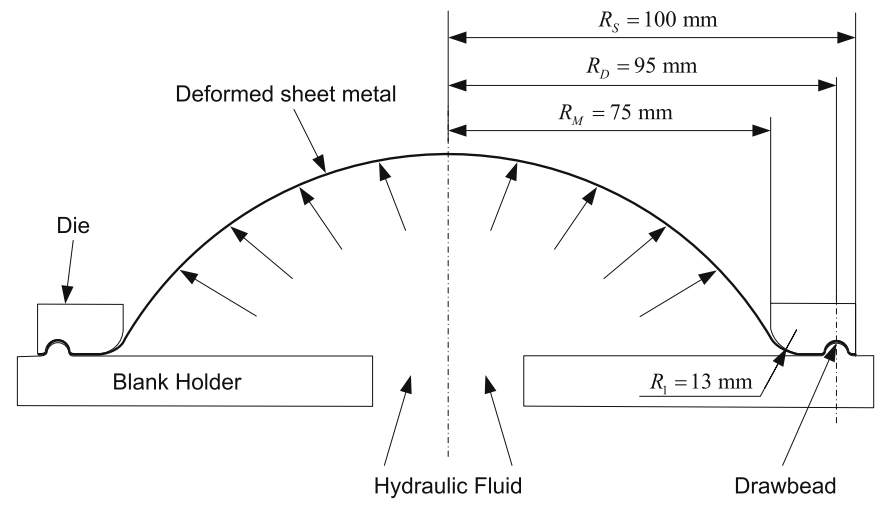

Fig. 1. Mechanical model of the bulge test, with the identification of the principal dimensions of the tool (Santos et al., 2010). (a)

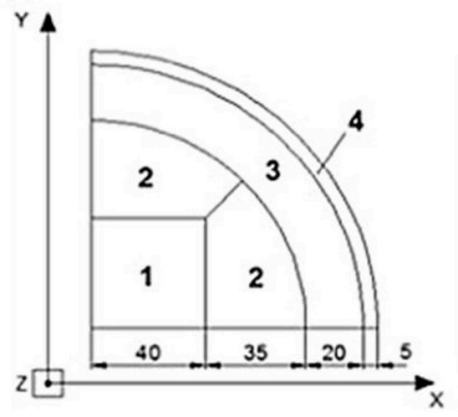

(b)

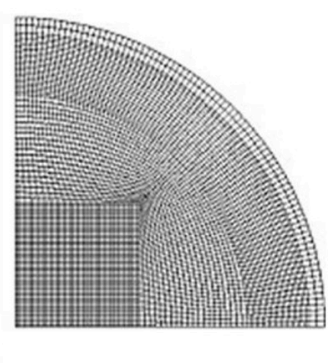

Fig. 2. Blank sheet discretization: (a) discretization zones with dimensions in mm; (b) finite elements mesh (Reis et al., 2017b).

$$
\begin{aligned}
& \frac{P_{i}^{N u m}\left(A_{j}+\delta A_{j}\right)-P_{i}^{N u m}\left(A_{j}\right)}{P_{i}^{N u m}\left(A_{j}\right)} \\
& =\frac{Y_{i}\left(A_{j}+\delta A_{j}, \bar{\varepsilon}_{i}\left(A_{j}+\delta A_{j}\right)\right)-Y_{i}\left(A_{j}, \bar{\varepsilon}_{i}\left(A_{j}\right)\right)}{Y_{i}\left(A_{j}, \bar{\varepsilon}_{i}\left(A_{j}\right)\right)},
\end{aligned}
$$

where $P_{i}^{N u m}\left(A_{j}\right)$ and $P_{i}^{N u m}\left(A_{j}+\delta A_{j}\right)$ are the pressure values at the pole height for the instant, $i$, of the test, respectively for the constitutive parameters $A_{j}$, and for the same constitutive parameter affected by a small incremental value, $\left(A_{j}+\delta A_{j}\right) ; \quad Y_{i}\left(A_{j}, \bar{\varepsilon}_{i}\left(A_{j}\right)\right) \quad$ and $Y_{i}\left(A_{j}+\delta A_{j}, \bar{\varepsilon}_{i}\left(A_{j}+\delta A_{j}\right)\right)$ are the corresponding flow stresses at the pole of the bulge test. These stresses can be analytically evaluated from the hardening law (equation (7)), provided that the equivalent plastic strains, $\bar{s}_{i}\left(A_{j}\right)$ and $\bar{\varepsilon}_{i}\left(A_{j}+\delta A_{j}\right)$, are known (as done in Fig. 3 (a)). Fig. 3 (b) is plotted assuming that $Y_{i}\left(A_{j}+\delta A_{j}, \bar{\varepsilon}_{i}\left(A_{j}+\delta A_{j}\right)\right)$ is equal to $Y_{i}\left(A_{j}+\delta A_{j}, \bar{\varepsilon}_{i}\left(A_{j}\right)\right)$. According to this figure, the relative difference in pressure $v s$. pole height and flow stress $v s$. pole height are still similar, as in Fig. 3 (a), and therefore the condition $Y_{i}\left(A_{j}+\delta A_{j}, \bar{\varepsilon}_{i}\left(A_{j}+\delta A_{j}\right)\right)=Y_{i}\left(A_{j}+\delta A_{j}, \bar{\varepsilon}_{i}\left(A_{j}\right)\right)$ can also be assumed in equation (10). A similar approximation has been considered in previous works (Bäker and Shrot, 2013; Endelt and Danckert, 2009), although under different types of analysis.

\subsection{Proposed approach}

Based on the sensitivity analysis previously undertaken, a procedure is developed to calculate an approximation for the Jacobian matrix. The sensitivity study mentioned above showed similarities between the relative difference in pressure and flow stress during the bulge test (see Fig. 3), which led to assume the equality expressed in equation (10). Generalizing this equation for other global variables (e.g. load), $g_{i}{ }^{N u m}$, and assuming that $Y_{i}\left(A_{j}+\delta A_{j}, \bar{\varepsilon}_{i}\left(A_{j}+\delta A_{j}\right)\right)=Y_{i}\left(A_{j}+\delta A_{j}, \bar{\varepsilon}_{i}\left(A_{j}\right)\right)$, equation (10) can be written as follows: 
Table 1

Constitutive parameters used for the sensitivity analysis of the circular bulge test results considering relative increments of $0.5 \%$ in each parameter.

\begin{tabular}{|c|c|c|c|c|c|c|c|c|}
\hline Designation & $Y_{0}[\mathrm{MPa}]$ & $C[\mathrm{MPa}]$ & $n$ & $F$ & $G$ & $H$ & $L=M$ & $N$ \\
\hline Reference Material (Teodosiu and Bouvier, 2001) & 330.30 & 1093.00 & 0.1870 & 0.5127 & 0.4975 & 0.5025 & 1.5000 & 1.2729 \\
\hline$\delta Y_{0}$ & 331.95 & 1093.00 & 0.1870 & & & & & \\
\hline$\delta C$ & 330.30 & 1098.47 & 0.1870 & & & & & \\
\hline$\delta n$ & 330.30 & 1093.00 & 0.1879 & & & & & \\
\hline
\end{tabular}
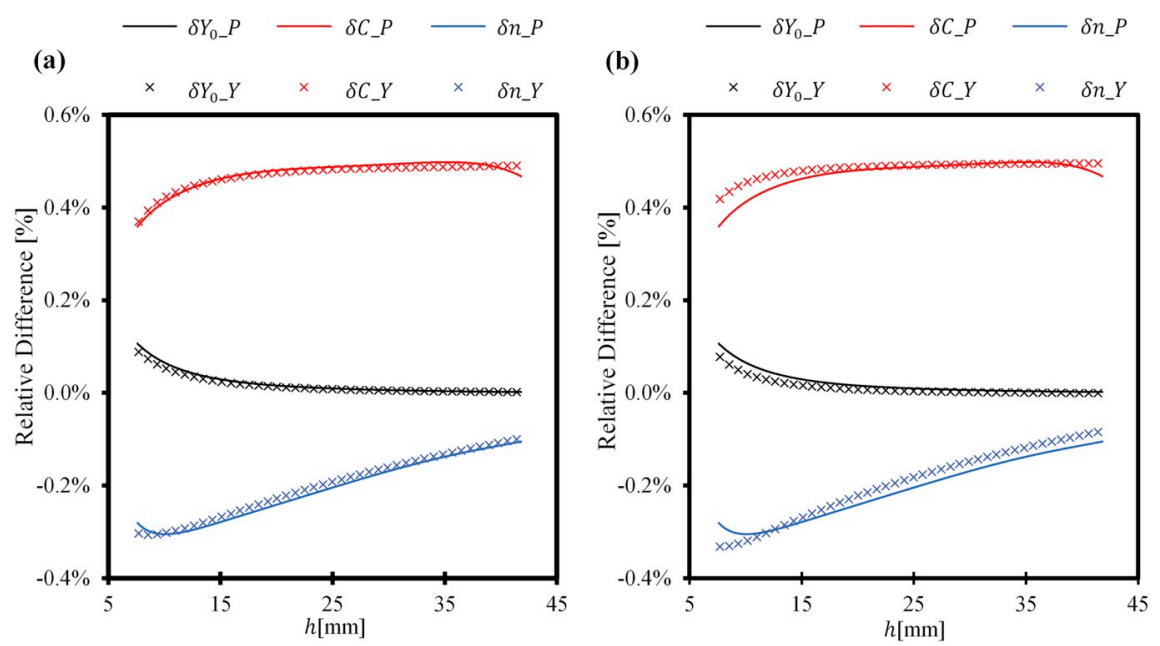

Fig. 3. Relative difference in pressure, “_$P$ ", and flow stress, “_$Y$ ”, for the cases " $\delta Y_{0}$ ”, " $\delta C$ " and " $\delta n$ ". The flow stresses values were determined as follows: (a) considering the exact values, i.e. taking into account that $Y_{i}\left(A_{j}+\delta A_{j}, \bar{\varepsilon}_{i}\left(A_{j}+\delta A_{j}\right)\right) \neq Y_{i}\left(A_{j}+\delta A_{j}, \bar{\varepsilon}_{i}\left(A_{j}\right)\right)$; (b) assuming that $Y_{i}\left(A_{j}+\delta A_{j}, \bar{\varepsilon}_{i}\left(A_{j}+\delta A_{j}\right)\right)=Y_{i}\left(A_{j}+\delta A_{j}, \bar{\varepsilon}_{i}\left(A_{j}\right)\right)$.

$\frac{g_{i}^{N u m}\left(A_{j}+\delta A_{j}\right)-g_{i}^{N u m}\left(A_{j}\right)}{g_{i}^{N u m}\left(A_{j}\right)}=\frac{Y_{i}\left(A_{j}+\delta A_{j}, \bar{\varepsilon}_{i}\left(A_{j}\right)\right)-Y_{i}\left(A_{j}, \bar{\varepsilon}_{i}\left(A_{j}\right)\right)}{Y_{i}\left(A_{j}, \bar{\varepsilon}_{i}\left(A_{j}\right)\right)}$.

Based in equation (11), equations (4) and (5) can be written, respectively:

$J_{i j}=\frac{g_{i}^{N u m}\left(A_{j}\right)}{Y_{i}\left(A_{j}, \bar{\varepsilon}_{i}\left(A_{j}\right)\right)} \frac{Y_{i}\left(A_{j}+\delta A_{j}, \bar{\varepsilon}_{i}\left(A_{j}\right)\right)-Y_{i}\left(A_{j}, \bar{\varepsilon}_{i}\left(A_{j}\right)\right)}{\delta A_{j}}$,

$J_{i j}=\frac{1}{Y_{i}\left(A_{j}, \bar{\varepsilon}_{i}\left(A_{j}\right)\right)} \frac{Y_{i}\left(A_{j}+\delta A_{j}, \bar{\varepsilon}_{i}\left(A_{j}\right)\right)-Y_{i}\left(A_{j}, \bar{\varepsilon}_{i}\left(A_{j}\right)\right)}{\delta A_{j}}$.

When $\delta A_{j}$ approaches zero, equations (12) and (13) can be respectively written as:

$J_{i j}=\frac{g_{i}^{N u m}\left(A_{j}\right)}{Y_{i}\left(A_{j}, \bar{\varepsilon}_{i}\left(A_{j}\right)\right)} \frac{\partial Y_{i}\left(A_{j}, \bar{\varepsilon}_{i}\left(A_{j}\right)\right)}{\partial A_{j}}$,

$J_{i j}=\frac{1}{Y_{i}\left(A_{j}, \bar{\varepsilon}_{i}\left(A_{j}\right)\right)} \frac{\partial Y_{i}\left(A_{j}, \bar{\varepsilon}_{i}\left(A_{j}\right)\right)}{\partial A_{j}}$.

The calculation of the terms of the Jacobian matrix by equations (14) or (15) requires performing one numerical simulation per iteration for obtaining $g_{i}{ }^{\text {Num }}\left(A_{j}\right)$ and the respective value of the equivalent plastic strain, $\bar{\varepsilon}_{i}\left(A_{j}\right)$, for calculating $Y_{i}\left(A_{j}, \bar{\varepsilon}_{i}\left(A_{j}\right)\right)$ and $\partial Y_{i}\left(A_{j}, \bar{\varepsilon}_{i}\left(A_{j}\right)\right) / \partial A_{j}$. The flow stress, $Y_{i}\left(A_{j}, \bar{\varepsilon}_{i}\left(A_{j}\right)\right)$, is given by the hardening law and the determination of $\partial Y_{i}\left(A_{j}, \bar{\varepsilon}_{i}\left(A_{j}\right)\right) / \partial A_{j}$ resorts to its partial derivatives, with respect to each constitutive parameter, $A_{j}$. In case of the Swift hardening law (equation (7)), the partial derivatives, $\partial Y_{i}\left(A_{j}, \bar{\varepsilon}_{i}\left(A_{j}\right)\right) / \partial A_{j}$, are given by:

$\frac{\partial Y_{i}\left(Y_{0}, \bar{\varepsilon}_{i}\left(Y_{0}\right)\right)}{\partial Y_{0}}=\left(\frac{Y_{0}}{C}\right)^{\frac{1}{n}-1}\left(\bar{\varepsilon}_{i}+\left(\frac{Y_{0}}{C}\right)^{\frac{1}{n}}\right)^{n-1}$,

$$
\begin{aligned}
\frac{\partial Y_{i}\left(C, \bar{\varepsilon}_{i}(C)\right)}{\partial C}= & \bar{\varepsilon}_{i}\left(\bar{\varepsilon}_{i}+\left(\frac{Y_{0}}{C}\right)^{\frac{1}{n}}\right)^{n-1}, \\
\frac{\partial Y_{i}\left(n, \bar{\varepsilon}_{i}(n)\right)}{\partial n}= & C\left(\ln \left(\bar{\varepsilon}_{i}+\left(\frac{Y_{0}}{C}\right)^{\frac{1}{n}}\right)\left(\bar{\varepsilon}_{i}+\left(\frac{Y_{0}}{C}\right)^{\frac{1}{n}}\right)^{n}\right. \\
& \left.-\ln \left(\frac{Y_{0}}{C}\right)\left(\frac{Y_{0}}{C}\right)^{\frac{1}{n}}\left(\bar{\varepsilon}_{i}+\left(\frac{Y_{0}}{C}\right)^{\frac{1}{n}}\right)^{n-1} n^{-1}\right),
\end{aligned}
$$

where $\bar{\varepsilon}_{i}$ is the equivalent plastic strain measured at the instant, $i$, of the test for the reference vector of constitutive parameters, $\mathbf{A}=\left[Y_{0}, C, n\right]$. The equivalent plastic strain is numerically obtained at a point of the specimen for which equation (11) is fulfilled. This can occur at distinct locations of the specimen. Fig. 4 shows the evolution of the relative difference in pressure and flows stress with the pole height, for the hardening parameters, $Y_{0}, C$ and $n$, at different locations of the specimen. The flow stress evolutions are shown for points located at the pole and at initial distances of 20, 40 and $60 \mathrm{~mm}$ from the pole, which are respectively designated by "\%Y_Pole", "\% $Y \_20 "$, " $\% Y_{-} 40$ " and "\% Y_60". In this figure, the results of the relative difference in pressure, "\%P", and flow stress at the pole of the bulge, "\%Y_Pole" are the same as those shown in Fig. 3 (b). The relative difference of pressure $v s$. pole height and of flow stress $v s$. pole height, evaluated at these points are still relatively close, with identical evolutions, enabling the assumption that equation (11) is suitable. Additionally, other points were successfully tested; only those located between the die and the blank holder, which have relatively small values of plastic strain, are exceptions. In the case of other mechanical tests, it is recommended to select a point within a region of relatively high equivalent plastic strain, in order to evaluate the sensitivity matrix up to high values of plastic deformation. In contrast, points with relatively small values of plastic strain should be avoided.

In summary, the proposed approach is valid when equation (11) is 


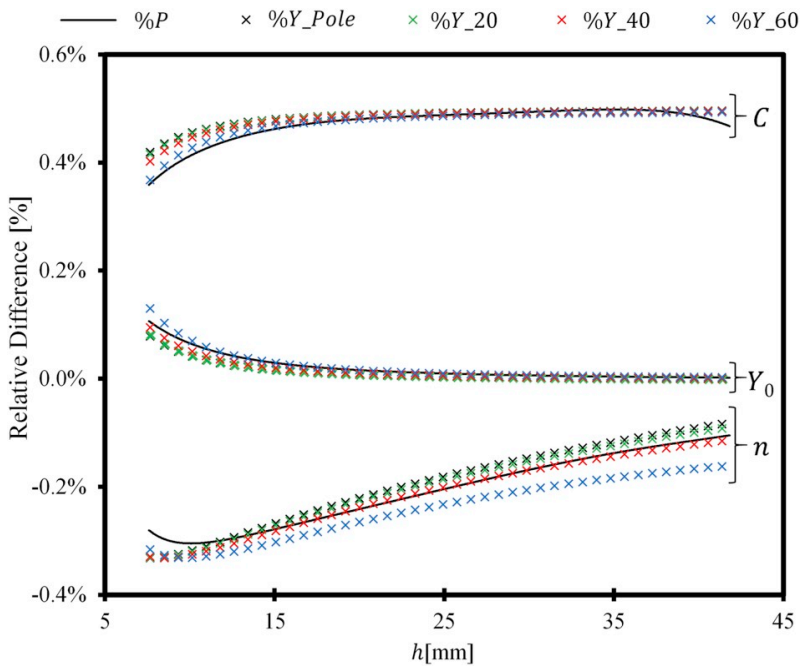

Fig. 4. Relative difference in pressure, " $\% P$ ", and in flow stress at the pole, "\% Y_Pole", and at points located at an initial distance of 20, 40 and $60 \mathrm{~mm}$ from the pole, which are respectively designated by "\% $Y \_20$ ", "\% $Y_{-} 40$ " and "\%Y_60".

verified under the assumption that the flow stress at the pole is not influenced by the variation of the equivalent plastic strain due to the incremental variation of the hardening law parameters, i.e. $Y_{i}\left(A_{j}+\delta A_{j}, \bar{\varepsilon}_{i}\left(A_{j}+\delta A_{j}\right)\right)=Y_{i}\left(A_{j}+\delta A_{j}, \bar{\varepsilon}_{i}\left(A_{j}\right)\right)$. In this case, the proposed approach allows the analytical determination of the terms of the work hardening Jacobian matrix using equations (14) or (15).

A similar sensitivity analysis was performed for the parameters of the Hill'48 yield criterion. Although the approximation presented in equation (10) is also verified for these parameters, the accurate determination of the terms of the Jacobian matrix (equations (14) and (15)) is no longer possible when the assumption $Y_{i}\left(A_{j}+\delta A_{j}, \bar{\varepsilon}_{i}\left(A_{j}+\delta A_{j}\right)\right)=Y_{i}\left(A_{j}+\delta A_{j}, \bar{\varepsilon}_{i}\left(A_{j}\right)\right)$ is considered. Nevertheless, when the parameters of the yield criterion and the hardening law are simultaneously optimized, the proposed approach can still be used to analytically compute the terms of the Jacobian matrix relative to the hardening parameters. At the same time, forward finite differences can be used to numerically compute the terms of the Jacobian matrix relative to the yield criterion parameters.

\section{Performance of the analytical approach for the Jacobian matrix}

In order to evaluate the performance of the proposed approach, three different inverse identification strategies were performed, concerning the use of Levenberg-Marquardt algorithm to determine the constitutive parameters of metal sheets. These inverse identification procedures, described in literature, are based on the following mechanical tests: (i) circular bulge test; (ii) cruciform tensile test; and (iii) reverse shear test. The data used in the inverse identifications are numerically generated using the constitutive parameters identified, for a DP600 steel, by Teodosiu and Bouvier (2001). The use of fictitious data allows the appropriate comparison between the identified and the reference results, i.e. without the errors associated with experimental measurements.

\subsection{Circular bulge test}

The circular bulge test is used to identify the work hardening behaviour described by the Swift law. The identification strategy consists of minimizing the gap between the reference and numerical curves, pressure $v s$. pole height, of the circular bulge test. The minimization is performed by using the Levenberg-Marquardt algorithm, with the following objective function, $f(\mathbf{A})$ :

$f(\mathbf{A})=\frac{1}{m} \sum_{i=1}^{m}\left(P^{\mathrm{Num}}\left(h_{i}\right)-P^{\operatorname{Ref}}\left(h_{i}\right)\right)^{2}$,

where $\mathbf{A}=\left[Y_{0}, C, n\right]$ is the vector of parameters to be optimized; $P^{\mathrm{Num}}\left(h_{i}\right)$ and $P^{\mathrm{Ref}}\left(h_{i}\right)$ are respectively the numerical and reference values of the pressure for a given value of pole height, $h_{i}$; and $m$ is the number of measuring instants. Polynomial interpolation is used to assess the numerical and reference pressure values for the same pole height, (see Reis et al. (2017a)). A total of 1000 measuring instants, uniformly distributed over the range of pole heights, were used. The parameters of the Hill' 48 criterion are kept fixed during the identification. The identification ends when the differences between the numerical and reference values of pressure are unnoticeable, which is consistent to $f(\mathbf{A}) \leq 10^{-4} \mathrm{MPa}$.

In order to illustrate the performance of the proposed approach, the work hardening parameters of the reference material (see Table 1) were identified. The Jacobian matrix was computed resorting to the proposed approach and via forward finite differences, with relative increments of $0.05 \%, 0.5 \%$ and $5 \%$ in each parameter, hereafter respectively designated by "FFD_0.05\%", "FFD_0.5\%" and "FFD_5\%". In case of the proposed approach, an interpolation is performed to enable the evaluation of the equivalent plastic strain for each pole height. The parameters used for the initial estimate and those identified are given in Table 2. The total number of iterations and numerical simulations used in the identification are also indicated. Whatever the strategy, the identified parameters are close to those of the reference material. In order to take a broad understanding of the differences between the identified and the reference parameters, Fig. 5 (a) compares the strategies in terms of work hardening curves. The relative differences in stress, between the results of any strategy and the reference material, have an average value less than $0.9 \%$. Fig. 5 (b) presents the evolution of the objective function value with the accumulated number of numerical simulations, showing that this number is lower for the proposed strategy. Although, finite differences require a lower number of iterations, the use of a higher number of numerical simulations per iteration make this approach computationally less efficient than the proposed approach, as can be observed in Fig. 5 (b). In summary, the use of the proposed strategy leads to a significant reduction in the total number of simulations; at least $44.4 \%$ when compared to the use of finite differences, without compromising the identification results.

For a broader comparison of the two types of approach, Fig. 6 shows the hardening parameters sensitivities as a function of the pole height, for the first iteration. For the forward finite-differences, the sensitivities

Table 2

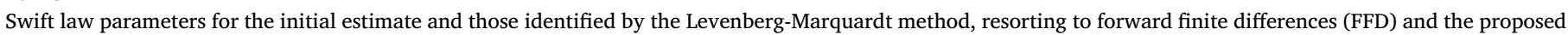
approach to compute the Jacobian matrix.

\begin{tabular}{|c|c|c|c|c|c|c|}
\hline Designation & $Y_{0}[\mathrm{MPa}]$ & $C[\mathrm{MPa}]$ & $n$ & $f(\mathbf{A})[\mathrm{MPa}]$ & Number of Iterations & Number of Simulations \\
\hline Initial Estimate & 200.00 & 980.25 & 0.300 & 5.11 & - & - \\
\hline FFD_0.05\% & 335.95 & 1092.45 & 0.186 & $5.54 \times 10^{-5}$ & 4 & 13 \\
\hline FFD_0.5\% & 330.71 & 1092.97 & 0.187 & $2.48 \times 10^{-7}$ & 4 & 13 \\
\hline FFD_5\% & 337.01 & 1091.65 & 0.186 & $4.66 \times 10^{-5}$ & 3 & 9 \\
\hline Proposed approach & 328.05 & 1094.90 & 0.187 & $5.50 \times 10^{-5}$ & 5 & 5 \\
\hline
\end{tabular}



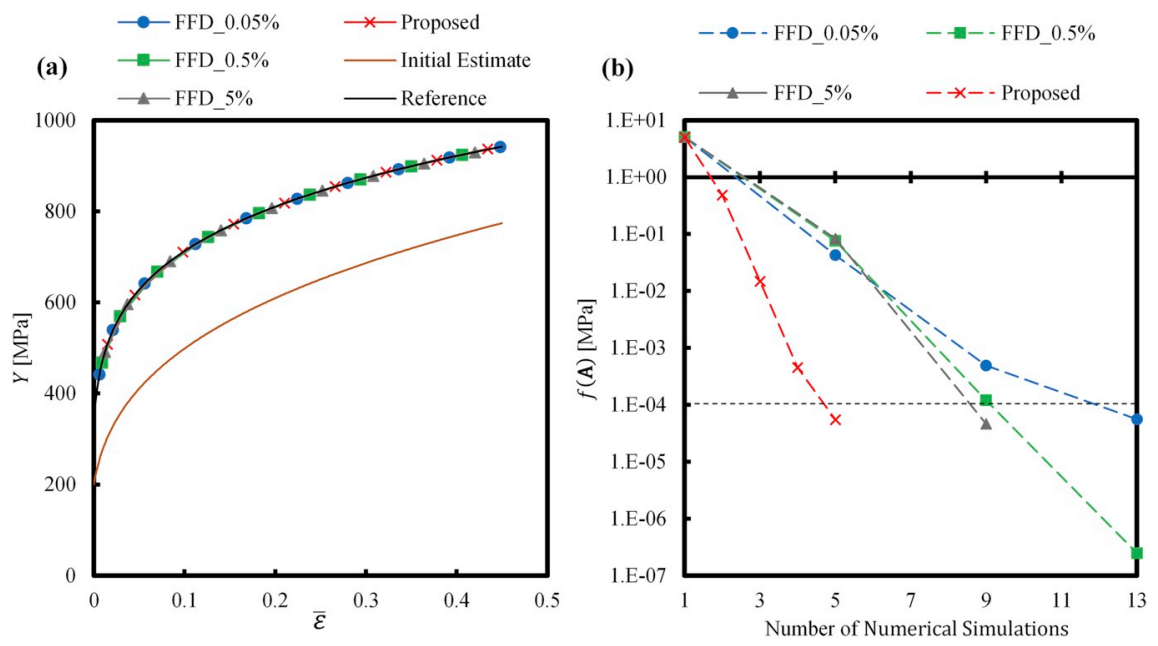

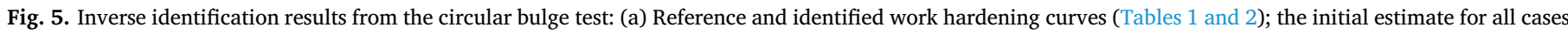
is also shown (Table 2); (b) Evolution of the objective function with the accumulated number of numerical simulations for the different cases.

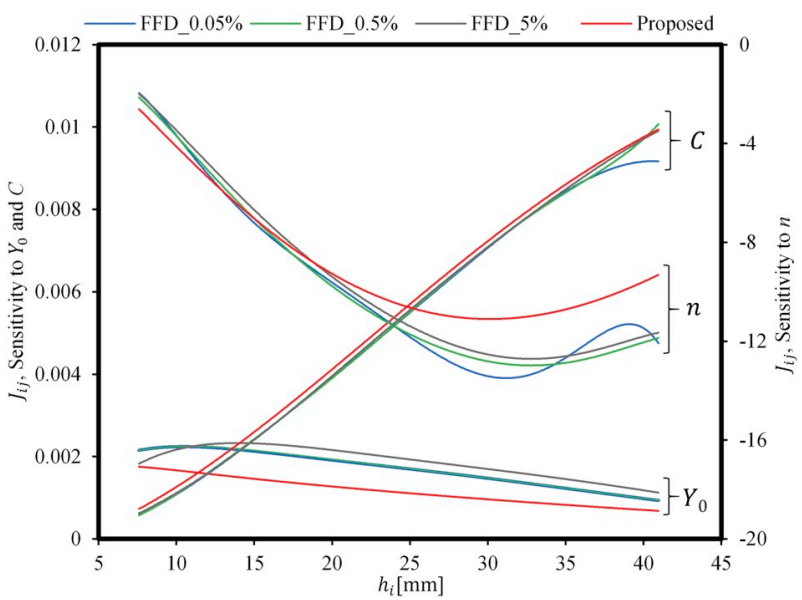

Fig. 6. Hardening parameters sensitivity as a function of the pole height: comparison between the proposed approach and the forward finite differences with increments of $0.05 \%, 0.5 \%$ and $5 \%$.

(equation (4)) are essentially similar whatever the value of the increment, although some differences can be observed for high values of pole height. These differences can be related to round off errors that arise when forward finite differences with small increments $(0.05 \%$ and $0.5 \%)$ are used. The sensitivities obtained with the proposed approach (equation (14)) present some discrepancies relatively to those from forward finite differences, as consequence of the assumptions made for the proposed approach. Nevertheless, the observed discrepancies lead to the similar identification result (see Fig. 5 (a)). The only consequence is the increase of the number of iterations while reducing the total number of numerical simulations.

The circular bulge test is now used to identify the parameters of the Swift law, assuming that the isotropic hardening behaviour of the reference material is modelled by a different hardening law, the Voce law (Voce, 1948). The purpose of this identification is to test the performance of the proposed methodology when the behaviour of the material is not perfectly described by the law whose parameters are intended to identify, and thus to analyse a case for which the identification errors will be greater than in the previous one. The Voce hardening law is written as follows:

$Y=Y_{0}+\left(Y_{S a t}-Y_{0}\right)\left(1-\exp \left(-C_{Y} \bar{\varepsilon}\right)\right)$,

where $Y_{0}, Y_{\text {Sat }}$ and $C_{Y}$ are the material parameters. Table 3 shows these parameters for the reference material, obtained by fitting points of the hardening curve plotted from the parameters of Table 1 . The parameters used for the initial estimate and those identified (via finite differences and using the proposed approach) are given in Table 4; the total number of iterations and numerical simulations used in the identifications are also indicated. The identification procedure was terminated when $f(\mathbf{A})$ stabilizes, which occurs for values of $f(\mathbf{A})$ close to $5 \times 10^{-3} \mathrm{MPa}$. Fig. 7 (a) shows the work hardening curves obtained using finite differences and the proposed approach, which overlap each other; the relative differences in stress between the identified work hardening curves and the reference material have an average value less than $1.67 \%$. Fig. 7 (b) shows that the total number of required numerical simulations is significantly lower for the proposed strategy, i.e. a reduction of $64.7 \%$ is observed when compared to the use of finite differences, without compromising the identification results.

\subsection{Cruciform test}

The cruciform test is now used to identify the Swift hardening law parameters based on the identification procedure proposed by Prates et al. (2014). The schematic representation of the cruciform specimen is shown in Fig. 8. During the test, equal displacements are imposed along the Ox and Oy axes on the border of the grips (dashed lines in Fig. 8). In this strategy, the optimization of the work hardening parameters is carried out by minimizing the gap between the numerical and reference load $v s$. displacement curves along the Ox and Oy axes. The LevenbergMarquardt method is used to minimize the following objective function, $f(\mathbf{A})$ :

$$
\begin{aligned}
f(\mathbf{A})= & \frac{1}{m}\left[\sum_{i=1}^{m}\left(\frac{R_{O x}^{N u m}\left(\Delta d_{i}\right)-R_{O x}^{R e f}\left(\Delta d_{i}\right)}{R_{O x}^{R e f}\left(\Delta d_{i}\right)}\right)^{2}\right. \\
& \left.+\sum_{i=1}^{m}\left(\frac{R_{O y}^{N u m}\left(\Delta d_{i}\right)-R_{O y}^{R e f}\left(\Delta d_{i}\right)}{R_{O y}^{R e f}\left(\Delta d_{i}\right)}\right)^{2}\right],
\end{aligned}
$$

where $\mathbf{A}=\left[Y_{0}, C, n, F\right]$ is the vector of parameters to be optimized. $F$ is a parameter of the Hill'48 criterion (equation (6)) that is simultaneously optimized with the hardening law parameters $\left(Y_{0}, C\right.$ and $\left.n\right)$, following the approach suggested by Prates et al. (2014); $R_{O x}^{N u m}\left(\Delta d_{i}\right)$ and $R_{O x}^{R e f}\left(\Delta d_{i}\right)$ are respectively the numerical and reference values of the load in the Ox axis; $R_{O y}^{N u m}\left(\Delta d_{i}\right)$ and $R_{O y}^{R e f}\left(\Delta d_{i}\right)$ are respectively the numerical and reference values of load in the Oy axes; these load values are measured for the imposed displacement, $\Delta d_{i}$, that is equal for points "A" (Ox axis) and "B" (Oy axis); $m$ is the total number of instants of measurement, uniformly distributed over the range of displacements (in 
Table 3

Constitutive parameters of the reference material modelled by the Voce hardening law.

\begin{tabular}{|c|c|c|c|c|c|c|c|c|}
\hline Designation & $Y_{0}[\mathrm{MPa}]$ & $Y_{\text {Sat }}[\mathrm{MPa}]$ & $C_{Y}$ & $F$ & $G$ & $H$ & $L=M$ & $N$ \\
\hline Reference material & 438.61 & 932.10 & 7.70 & 0.5127 & 0.4975 & 0.5025 & 1.5000 & 1.2729 \\
\hline
\end{tabular}

Table 4

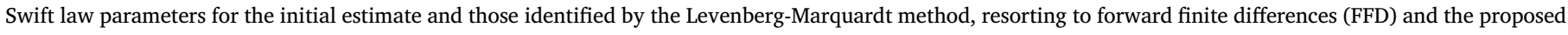
approach to compute the Jacobian matrix. The reference material follows the Voce hardening law (see Table 3).

\begin{tabular}{|c|c|c|c|c|c|c|}
\hline Designation & $Y_{0}[\mathrm{MPa}]$ & $C[\mathrm{MPa}]$ & $n$ & $f(\mathbf{A})[\mathrm{MPa}]$ & Number of Iterations & Number of Simulations \\
\hline Initial Estimate & 200.00 & 980.25 & 0.300 & 5.40 & - & - \\
\hline FFD_0.05\% & 335.19 & 1147.73 & 0.214 & $5.11 \times 10^{-3}$ & 5 & 17 \\
\hline FFD_0.5\% & 367.21 & 1164.43 & 0.224 & $4.66 \times 10^{-3}$ & 5 & 17 \\
\hline FFD_5\% & 358.69 & 1160.74 & 0.222 & $4.66 \times 10^{-3}$ & 5 & 17 \\
\hline Proposed approach & 371.25 & 1165.46 & 0.225 & $4.70 \times 10^{-3}$ & 6 & 6 \\
\hline
\end{tabular}

this work, $m=100$ ).

The equivalent plastic strains were evaluated in the middle of the arms of the cruciform specimen, points "C" and "D" of Fig. 8, where the equivalent plastic strain is relatively high. For these points, equation (11) was verified and thus the proposed strategy can be applied. However, in case of the parameter $F$ the assumption $Y_{i}\left(A_{j}+\delta A_{j}, \bar{\varepsilon}_{i}\left(A_{j}+\delta A_{j}\right)\right)=Y_{i}\left(A_{j}+\delta A_{j}, \bar{\varepsilon}_{i}\left(A_{j}\right)\right)$ (taken into account on equation (11)) is no longer acceptable. Therefore, the sensitivity of parameter $F$ of the Hill' 48 criterion is evaluated using finite differences. The identification procedures ended when the differences between the numerical and reference values of load are imperceptible, which is compatible to $f(\mathbf{A}) \leq 10^{-6}$.

The results of the inverse identifications of the reference material (Table 1), using the proposed approach and forward finite differences, are shown in Table 5 and Fig. 9. Also in this test, both types of strategies provided parameters close to those of the reference material, such that the relative differences in stress has an average value less than $0.21 \%$. The identifications resorting to the proposed strategy required 5 iterations, while finite differences involved 4 iterations due to the better estimation of the Jacobian matrix. Nevertheless, the use of the proposed strategy leads to a significant reduction of $43.8 \%$ in the total number of numerical simulations.

\subsection{Reverse shear test}

In this section, the reverse shear test is used to identify the isotropic and kinematic hardening laws parameters based on the identification

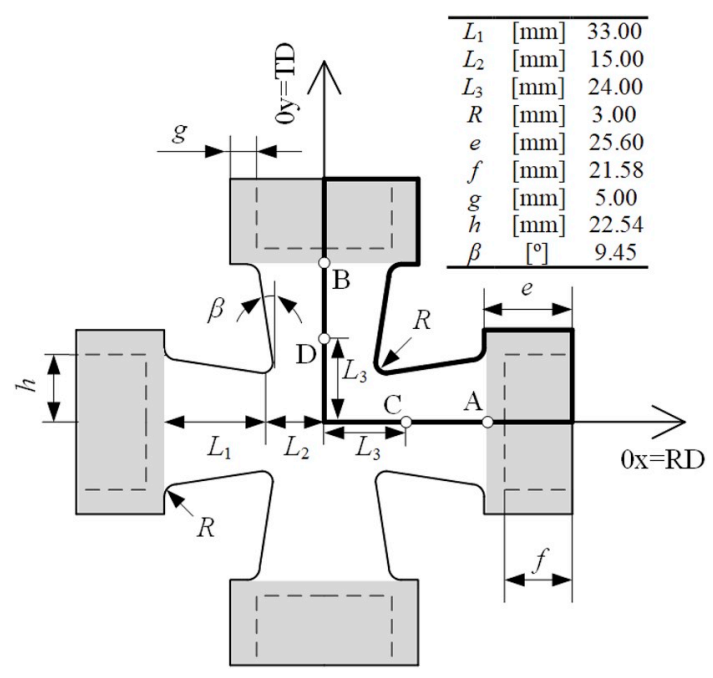

Fig. 8. Geometry and dimensions of the cruciform specimen. Adapted from Prates et al. (2014).

procedure proposed by Pereira et al. (2015). Fig. 10 shows the schematic representation of the shear notched specimen, as proposed by the authors. The numerical simulations are performed by imposing a displacement along the length direction on points located on the lateral surfaces that contains point A (Fig. 10) while the opposite surface is
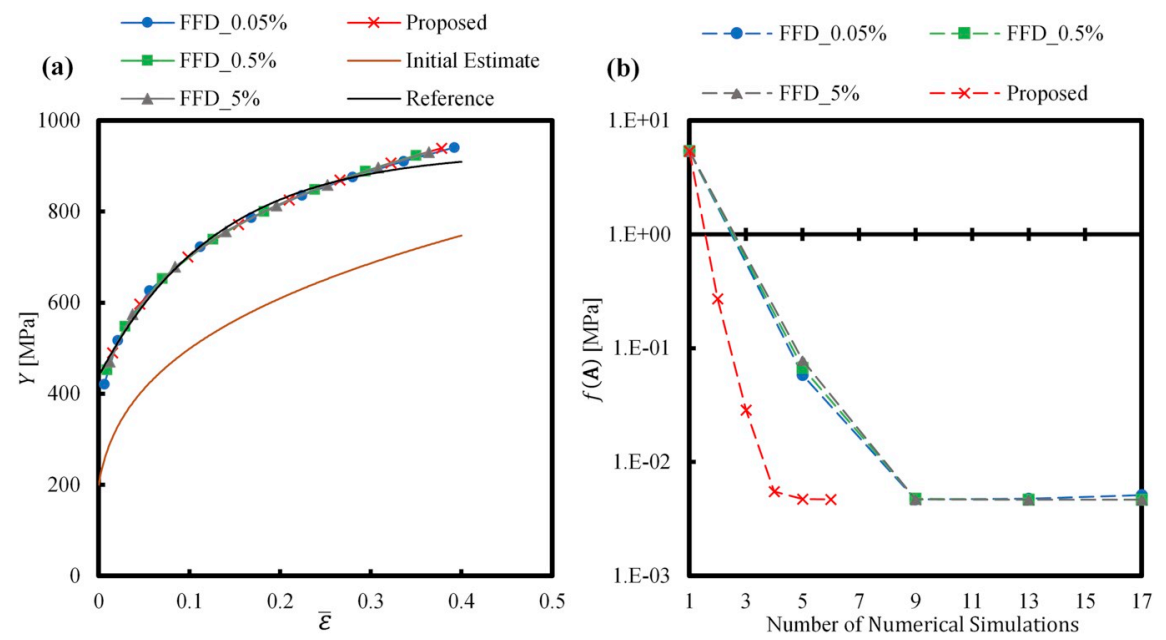

Fig. 7. Inverse identification results from the bulge test: (a) Reference (Voce) and identified (Swift) work hardening curves (Tables 3 and 4); the initial estimate for all cases is also shown (Table 4); (b) Evolution of the objective function with the accumulated number of numerical simulations for the different cases. 
Table 5

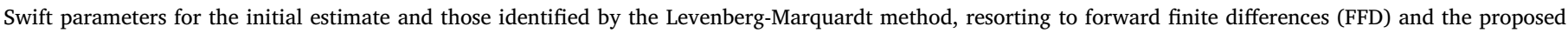
approach to compute the Jacobian matrix.

\begin{tabular}{|c|c|c|c|c|c|c|c|}
\hline Designation & $Y_{0}[\mathrm{MPa}]$ & $C[\mathrm{MPa}]$ & $n$ & $F$ & $f(\mathbf{A})$ & Number of Iterations & Number of Simulations \\
\hline Initial Estimate & 200.00 & 980.25 & 0.300 & 0.500 & $2.31 \times 10^{-1}$ & - & - \\
\hline FFD_0.05\% & 330.15 & 1094.44 & 0.187 & 0.187 & $1.65 \times 10^{-7}$ & 4 & 16 \\
\hline FFD_0.5\% & 330.12 & 1094.42 & 0.186 & 0.186 & $1.65 \times 10^{-7}$ & 4 & 16 \\
\hline FFD_5\% & 330.04 & 1094.43 & 0.186 & 0.186 & $1.68 \times 10^{-7}$ & 4 & 16 \\
\hline Proposed approach & 330.64 & 1094.30 & 0.186 & 0.186 & $1.91 \times 10^{-7}$ & 5 & 9 \\
\hline
\end{tabular}

fixed (Pereira et al., 2015). The identification of the isotropic and kinematic hardening parameters is carried out by minimizing the difference between the numerical and reference curves, load $v s$. displacement, along the loading and reverse paths. The Levenberg-Marquardt method was used to minimize the following objective function, $f(\mathbf{A})$ :

$$
\begin{aligned}
f(\mathbf{A})= & \frac{1}{m} \sum_{i=1}^{m}\left(\frac{R_{L}^{N u m}\left(\Delta d_{i}\right)-R_{L}^{R e f}\left(\Delta d_{i}\right)}{R_{L}^{R e f}\left(\Delta d_{i}\right)}\right)^{2} \\
& +\frac{1}{r} \sum_{i=1}^{r}\left(\frac{R_{R L}^{N u m}\left(\Delta d_{i}\right)-R_{R L}^{R e f}\left(\Delta d_{i}\right)}{R_{R L}^{R e f}\left(\Delta d_{i}\right)}\right)^{2},
\end{aligned}
$$

where $\mathbf{A}=\left[Y_{0}, C n, X_{S a t}, C_{X}\right]$ is the vector of parameters to be optimized (see equations (7) and (8)); $R_{L}^{\text {Num }}\left(\Delta d_{i}\right)$ and $R_{L}^{R e f}\left(\Delta d_{i}\right)$ are respectively the numerical and reference values of the load for a given displacement, $\Delta d_{i}$, along the loading path; $R_{R L}^{N u m}\left(\Delta d_{i}\right)$ and $R_{R L}^{R e f}\left(\Delta d_{i}\right)$ are respectively the numerical and reference values of the load along the reverse path; $m$ and $r$ are the total number of measuring instants along the loading and reverse paths, respectively. A total of 100 measuring instants along the range of displacements were used for each path. The displacement is measured in the edge of the gauge zone, for instance at point A in Fig. 10; the loading reversal is imposed after a displacement of $0.34 \mathrm{~mm}$. The identification ended when the differences between the numerical and reference values of load are unnoticeable, which is compatible to $f(\mathbf{A}) \leq 10^{-5}$.

In the previous examples of bulge and cruciform tests, the equality defined by equation (11) was used to evaluate the Jacobian matrix. In case of reverse shear test, the stress $Y_{i}$, in equation (11), should take into account the kinematic and isotropic hardening effects. For this purpose, the stress $Y_{i}$ is replaced by $Y_{i}^{*}$ in the following equations. The terms of the Jacobian matrix are now defined by the following equations, respectively, for the case of the residuals being expressed in terms of absolute or relative differences:

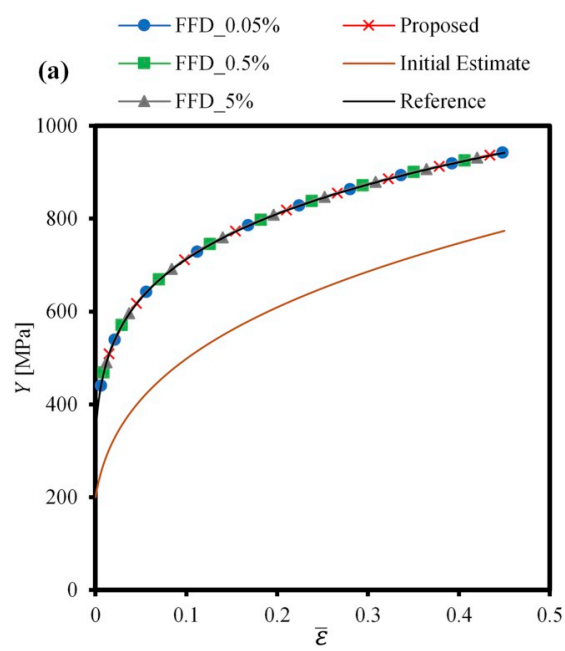

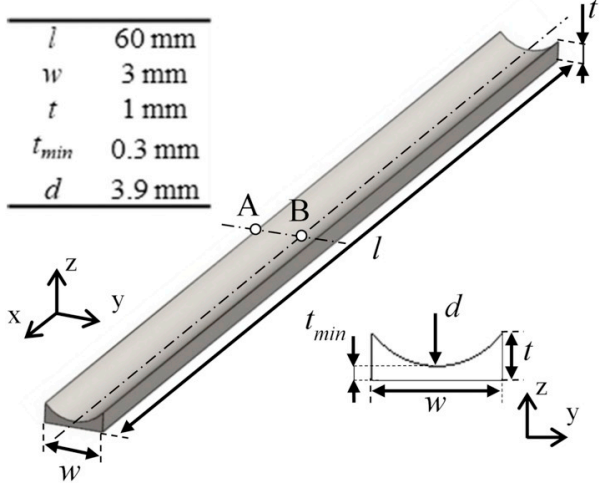

Fig. 10. Gauge area dimensions of the reverse shear test. Adapted from Pereira et al. (2015).

$J_{i j}=\frac{g_{i}{ }^{\text {Num }}\left(A_{j}\right)}{Y_{i}^{*}\left(A_{j}, \bar{\varepsilon}_{i}\left(A_{j}\right)\right)} \frac{\partial Y_{i}^{*}\left(A_{j}, \bar{\varepsilon}_{i}\left(A_{j}\right)\right)}{\partial A_{j}}$,

$J_{i j}=\frac{1}{Y_{i}^{*}\left(A_{j}, \bar{\varepsilon}_{i}\left(A_{j}\right)\right)} \frac{\partial Y_{i}^{*}\left(A_{j}, \bar{\varepsilon}_{i}\left(A_{j}\right)\right)}{\partial A_{j}}$,

where $Y_{i}^{*}\left(A_{j}, \bar{\varepsilon}_{i}\left(A_{j}\right)\right)$ is the stress for the constitutive parameters $A_{j}$.

In case of one dimensional loading with reversal, equation (8) can be analytically integrated to obtain the back-stress along the loading paths, $X^{\mathrm{L}}$, and the reverse loading paths $X^{\mathrm{RL}}$, respectively (Chiang, 2008):

$X^{L}=X_{S a t}+\left(X_{0}^{L}-X_{S a t}\right) \exp \left(-C_{X}\left(\bar{\varepsilon}-\bar{\varepsilon}_{0}^{L}\right)\right)$

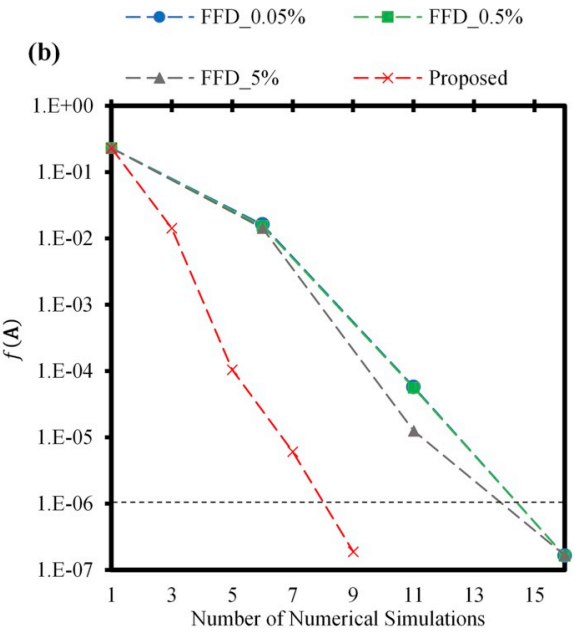

Fig. 9. Inverse identification results from the cruciform test: (a) Reference and identified work hardening curves (Tables 1 and 5); the initial estimate for all cases is also shown (Table 5); (b) Evolution of the objective function with the accumulated number of numerical simulations for the different cases. 
$X^{\mathrm{RL}}=-X_{\text {Sat }}+\left(X_{0}^{R L}+X_{\text {Sat }}\right) \exp \left(-C_{X}\left(\bar{\varepsilon}-\bar{\varepsilon}_{0}^{R L}\right)\right)$,

where $X_{0}^{L}$ and $\bar{\varepsilon}_{0}^{L}$ are respectively the back-stress and the equivalent plastic strain at the beginning of the loading paths $\left(X_{0}^{L}\right.$ and $\bar{\varepsilon}_{0}^{L}$ are equal to zero for the first loading path); $X_{0}^{R L}$ and $\bar{\varepsilon}_{0}^{R L}$ are respectively the backstress and the equivalent plastic strain at the beginning of the reverse loading paths.

Taking into account equations (7), (25) and (26) (knowing that $X_{0}^{R L}=X^{\mathrm{L}}\left(\bar{\varepsilon}=\bar{\varepsilon}_{0}^{R L}\right)$ as well as $\left.X_{0}^{L}=\bar{\varepsilon}_{0}^{L}=0\right)$, the stress $Y_{i}^{*}\left(A_{j}, \bar{\varepsilon}_{i}\left(A_{j}\right)\right)$ in the loading path, $Y_{i}^{*}\left(A_{j}, \bar{\varepsilon}_{i}\left(A_{j}\right)\right)^{L}$, and reverse path, $Y_{i}^{*}\left(A_{j}, \bar{\varepsilon}_{i}\left(A_{j}\right)\right)^{R L}$, are respectively given by:

$$
\begin{aligned}
Y_{i}^{*}\left(A_{j}, \bar{\varepsilon}_{i}\left(A_{j}\right)\right)^{L}= & Y+X^{\mathrm{L}}=C\left(\bar{\varepsilon}_{i}+\varepsilon_{0}\right)^{n}+X_{\text {Sat }}\left(1-\exp \left(-C_{X} \bar{\varepsilon}_{i}\right)\right), \\
Y_{i}^{*}\left(A_{j}, \bar{\varepsilon}_{i}\left(A_{j}\right)\right)^{R L}= & -Y+X^{\mathrm{RL}}=-C\left(\bar{\varepsilon}_{i}+\varepsilon_{0}\right)^{n}+X_{\text {Sat }}\left(1-\exp \left(-C_{X} \bar{\varepsilon}_{i}\right)\right) \\
& -2 X_{\text {Sat }}\left(1-\exp \left(-C_{X}\left(\bar{\varepsilon}_{i}-\bar{\varepsilon}_{0}^{R L}\right)\right)\right) .
\end{aligned}
$$

The partial derivatives of $Y_{i}^{*}\left(A_{j}, \bar{\varepsilon}_{i}\left(A_{j}\right)\right)$ with respect to each constitutive parameter of the hardening laws, are given by:

$$
\begin{aligned}
& \frac{\partial Y_{i}^{*}\left(Y_{0}, \bar{\varepsilon}_{i}\left(Y_{0}\right)\right)^{L}}{\partial Y_{0}}=-\frac{\partial Y_{i}^{*}\left(Y_{0}, \bar{\varepsilon}_{i}\left(Y_{0}\right)\right)^{R L}}{\partial Y_{0}}=\frac{\partial Y_{i}\left(Y_{0}, \bar{\varepsilon}_{i}\left(Y_{0}\right)\right)}{\partial Y_{0}} \\
& \frac{\partial Y_{i}^{*}\left(C, \bar{\varepsilon}_{i}(C)\right)^{L}}{\partial C}=-\frac{\partial Y_{i}^{*}\left(C, \bar{\varepsilon}_{i}(C)\right)^{R L}}{\partial C}=\frac{\partial Y_{i}\left(C, \bar{\varepsilon}_{i}(C)\right)}{\partial C} \\
& \frac{\partial Y_{i}^{*}\left(n, \bar{\varepsilon}_{i}(n)\right)^{L}}{\partial n}=-\frac{\partial Y_{i}^{*}\left(n, \bar{\varepsilon}_{i}(n)\right)^{R L}}{\partial n}=\frac{\partial Y_{i}\left(n, \bar{\varepsilon}_{i}(n)\right)}{\partial n} \\
& \frac{\partial Y_{i}^{*}\left(X_{\text {Sat }}, \bar{\varepsilon}_{i}\left(X_{\text {Sat }}\right)\right)^{L}}{\partial X_{\text {Sat }}}=1-\exp \left(-C_{X} \bar{\varepsilon}_{i}\right) \\
& \frac{\partial Y_{i}^{*}\left(X_{\text {Sat }}, \bar{\varepsilon}_{i}\left(X_{\text {Sat }}\right)\right)^{R L}}{\partial X_{\text {Sat }}}=1-\exp \left(-C_{X} \bar{\varepsilon}_{i}\right)-2\left(1-\exp \left(-C_{X}\left(\bar{\varepsilon}_{i}-\bar{\varepsilon}_{0}^{R L}\right)\right)\right)
\end{aligned}
$$

$\frac{\partial Y_{i}^{*}\left(C_{X}, \bar{\varepsilon}_{i}\left(C_{X}\right)\right)^{L}}{\partial C_{X}}=X_{S a t} \bar{\varepsilon}_{i} \exp \left(-C_{X} \bar{\varepsilon}_{i}\right)$,

$$
\begin{aligned}
\frac{\partial Y_{i}^{*}\left(C_{X}, \bar{\varepsilon}_{i}\left(C_{X}\right)\right)^{R L}}{\partial C_{X}}= & X_{S a t} \bar{\varepsilon}_{i} \exp \left(-C_{X} \bar{\varepsilon}_{i}\right) \\
& -2 X_{S a t}\left(\bar{\varepsilon}_{i}-\bar{\varepsilon}_{0}^{R L}\right) \exp \left(-C_{X}\left(\bar{\varepsilon}_{i}-\bar{\varepsilon}_{0}^{R L}\right)\right),
\end{aligned}
$$

where $\partial Y_{i}\left(Y_{0}, \bar{\varepsilon}_{i}\left(Y_{0}\right)\right) / \partial Y_{0}, \partial Y_{i}\left(C, \bar{\varepsilon}_{i}(C)\right) / \partial C$ and $\partial Y_{i}\left(n, \bar{\varepsilon}_{i}(n)\right) / \partial n$ are given by (16), (17) and (18), respectively; $\bar{\varepsilon}_{i}$ is the equivalent plastic strain measured at a point in the middle of the shear specimen, for instance point B in Fig. 10, where the equivalent plastic strain is relatively high. For this point, equation (11) was verified assuming that the flow stress $Y_{i}$ is now replaced by $Y_{i}^{*}$.

In order to test the proposed strategy, the procedure proposed by Pereira et al. (2015) is used to identify the parameters of the isotropic and kinematic hardening laws for the reference material presented in Table 6 . The inverse identification results using the proposed approach and the forward finite differences for the reference material are shown in Table 7 and the correspondent curves in Fig. 11 and Fig. 12; the initial estimates for the isotropic and kinematic laws used in the inverse identification are also indicated in this table. Both type of strategies provided parameters close to those of the reference material, such that the relative differences in equivalent stress and back-stress has an average value less than $0.46 \%$ and $1.12 \%$, respectively. The identifications resorting to the proposed strategy and forward finite differences with increments of $0.05 \%$ and $0.5 \%$ required 5 iterations, while with an increment of $0.5 \%$ involved 4 iterations. In summary, the use of the proposed strategy lead to a significant reduction in the required number of simulations, at least $73.7 \%$ when compared to forward finite differences, without compromising the inverse identification results.

\subsection{Final remarks}

The proposed strategy proved to be an expeditious alternative to the use of finite differences for computing the Jacobian matrix, in the context of the inverse analysis methodologies studied above. Based on the underlying assumptions, the framework of application of the proposed analytical estimate of the sensitivity matrix can be described as follows:

- parameters - identification of isotropic and kinematic hardening laws parameters of metal sheets, such as those considered in this work, respectively the Swift and Lemaitre-Chaboche laws, but also of other hardening laws whose application can be explored;

- experiments - when the experimental test imposes conditions such that the relationship between the load and the local stress is dictated by the equilibrium rather than from the mechanical behaviour of the material; such are the cases of the examples presented herein, but others involving for example torsion or bending can also be considered. It should be noted that, for the kinematic hardening, equations (25) and (26) have been developed for experiments that impose loading in one direction followed by reversing in the opposite direction;

- experimental measurements - the objective function should be defined using the evolution during the experimental test of a global variable, such as load, pressure, torsional or bending moment, which is directly related to the stress;

- variable for estimating the derivatives - a material point should be selected to numerically evaluate the equivalent plastic strain, within a region with a relatively high deformation, enabling the analytical calculus of the stress derivatives; these derivatives replace those of the global variable used in the objective function.

In case of inverse analysis methodologies involving for example other tests and/or global variables it is advisable the prior verification of the equation (11). In this context, the basic steps of this approach, which include the test of equation (11), can be summarized as follows:

1 Identify the global variables results used in the objective function (e.g. load or pressure $v$ s. displacement; torsional or bending moment $v s$. rotation or bending angle).

2 Define the analytical formulation (to be derived) for the stress, which consists of the isotropic hardening law (e.g. equation (7)) or, in case where kinematic hardening occurs, consists of adding or subtracting the isotropic and kinematic hardening laws, in direct and reverse paths respectively (e.g. equations (25) and (26)); in both cases the analytical formulation for the stress contains the hardening parameters to be optimized.

3 Analytically evaluate the stresses $Y_{i}\left(A_{j}, \bar{\varepsilon}_{i}\left(A_{j}\right)\right)$ and $Y_{i}\left(A_{j}+\delta A_{j}, \bar{\varepsilon}_{i}\left(A_{j}\right)\right)$ for a point in the specimen with a significant value of plastic deformation, where $\bar{\varepsilon}_{i}\left(A_{j}\right)$ is determined for the reference numerical simulation.

4 Test equation (11), by comparing the relative difference in the global variable (e.g. load, pressure, moment), obtained by numerical simulations, with the relative difference in stress (step 3).

5 Evaluate the sensitivity matrix using equations (14) or (15).

Table 6

Constitutive parameters of the reference material, taking into account isotropic and kinematic hardening (Teodosiu and Bouvier, 2001).

\begin{tabular}{llllllllll}
\hline Designation & $Y_{0}[\mathrm{MPa}]$ & $C[\mathrm{MPa}]$ & $n$ & $X_{\text {Sat }}[\mathrm{MPa}]$ & $C_{X}$ & $F$ & $G$ & $H$ & $L=M$ \\
\hline Reference material & 308.30 & 790.20 & 0.132 & 169.20 & 15.80 & \multirow{2}{*}{0.5127} & 0.4975 & 0.5025 & 1.5000 \\
\hline
\end{tabular}


Table 7

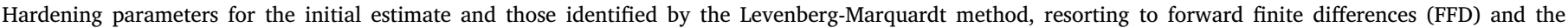
proposed approach to compute the Jacobian matrix.

\begin{tabular}{|c|c|c|c|c|c|c|c|c|}
\hline Designation & $Y_{0}[\mathrm{MPa}]$ & $C[\mathrm{MPa}]$ & $n$ & $X_{\text {Sat }}[\mathrm{MPa}]$ & $C_{X}$ & $f(\mathbf{A})$ & Number of Iterations & Number of Simulations \\
\hline Initial Estimate & 263.66 & 821.26 & 0.292 & 100.00 & 10.00 & $6.30 \times 10^{-2}$ & - & - \\
\hline FFD_0.05\% & 321.58 & 798.63 & 0.136 & 169.41 & 15.14 & $5.82 \times 10^{-6}$ & 5 & 25 \\
\hline FFD_0.5\% & 324.77 & 792.18 & 0.133 & 169.76 & 15.74 & $4.40 \times 10^{-6}$ & 4 & 19 \\
\hline FFD_5\% & 321.35 & 790.54 & 0.132 & 169.10 & 15.91 & $7.08 \times 10^{-6}$ & 5 & 25 \\
\hline Proposed approach & 308.04 & 790.34 & 0.132 & 166.54 & 16.33 & $7.29 \times 10^{-6}$ & 5 & 5 \\
\hline
\end{tabular}
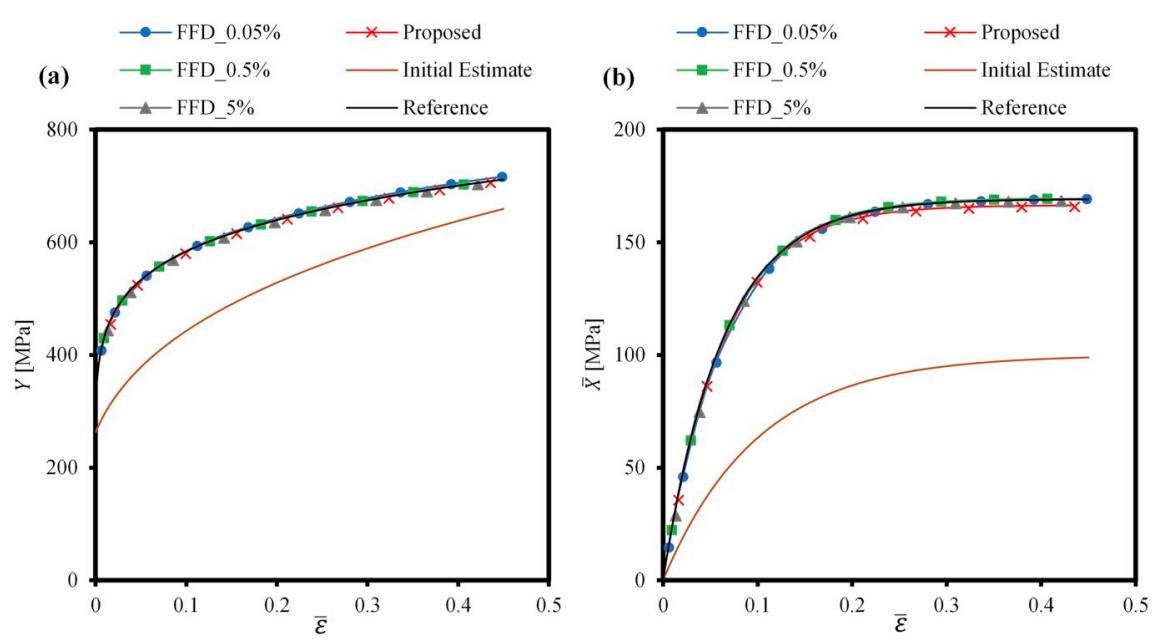

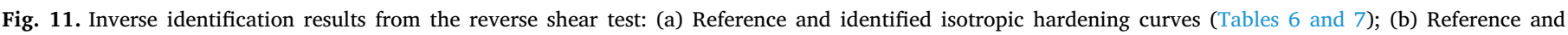
identified kinematic hardening curves (equation (9)) (Tables 6 and 7). The initial estimate for all cases is also shown (Table 7).

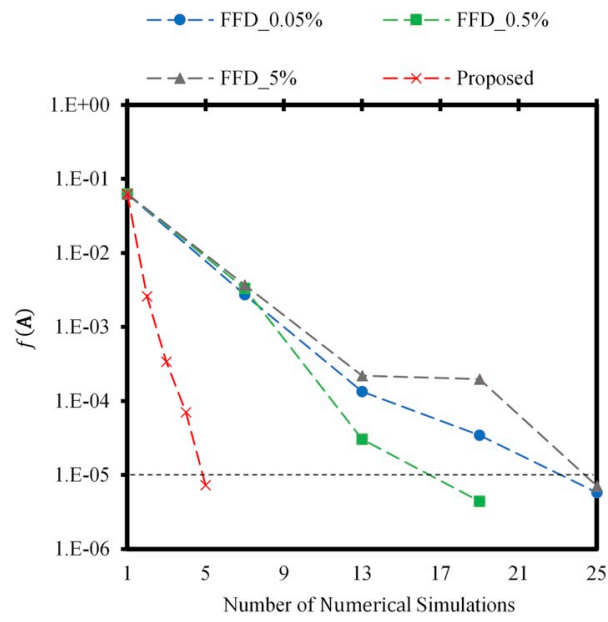

Fig. 12. Evolution of the objective function with the accumulated number of numerical simulations for the different approaches for the reverse shear test.

\section{Conclusions}

This work proposes a simple way to compute the Jacobian matrix for gradient-based optimization algorithms, based on the analytic differentiation of the isotropic and kinematic hardening laws of metal sheets. The proposed strategy was tested by performing inverse identification strategies using three different mechanical tests, the circular bulge, biaxial tensile, and reverse shear tests. It proved to be accurate in computing the sensitivity of global variables for these tests, which involves heterogeneous deformation. The main advantage of the proposed approach is the expeditious calculation of the Jacobian matrix, since it does not require performing the additional numerical simulations for each parameter, as for finite differences. When compared to other analytical approaches, the current one only requires the study of the evolution of the equivalent plastic strain in one or two points, thus avoiding the use of large amounts of data and the need to implement subroutines in FE codes. Although the proposed strategy is not suitable for computing the sensitivities of the numerical results to the yield criterion parameters, it can be complemented with the use of finitedifferences (or other strategy) for these parameters, reducing the total number of numerical simulations required to perform the identification procedure. The strategy applicability can be explored within the context of other mechanical tests/identification procedures/results.

\section{Acknowledgements}

This work was supported by funds from the Portuguese Foundation for Science and Technology and by FEDER funds via project reference UID/EMS/00285/2013. It was also supported by the projects: SAFEFORMING, co-funded by the Portuguese National Innovation Agency, by FEDER, through the program Portugal-2020 (PT2020), and by POCI, with reference POCI-01-0247-FEDER-017762; RDFORMING co-funded by Portuguese Foundation for Science and Technology, by FEDER, through the program Portugal-2020 (PT2020), and by POCI, with reference POCI-01-0145-FEDER-031243; EZ-SHEET co-funded by Portuguese Foundation for Science and Technology, by FEDER, through the program Portugal-2020 (PT2020), and by POCI, with reference POCI-01-0145-FEDER-031216. Two of the authors, A. F. G. Pereira and P.A. Prates, were supported by grants for scientific research from the Portuguese Foundation for Science and Technology (reference: SFRH/ BD/102519/2014 and SFRH/BPD/101465/2014, respectively). All supports are gratefully acknowledged.

\section{References}

Avril, S., Bonnet, M., Bretelle, A.-S., et al., 2008. Overview of identification methods of mechanical parameters based on full-field measurements. Exp. Mech. 48, 381-402. 
https://doi.org/10.1007/s11340-008-9148-y.

Bäker, M., Shrot, A., 2013. Inverse parameter identification with finite element simulations using knowledge-based descriptors. Comput. Mater. Sci. 69, 128-136. https:// doi.org/10.1016/j.commatsci.2012.11.059.

Chaboche, J.L., 1989. Constitutive equations for cyclic plasticity and cyclic viscoplasticity. Int. J. Plast. 5, 247-302. https://doi.org/10.1016/0749-6419(89)90015-6.

Chiang, D.-Y., 2008. Modelling and characterization of cyclic relaxation and ratcheting using the distributed-element model. Appl. Math. Model. 32, 501-513. https://doi. org/10.1016/j.apm.2007.01.002.

Cooreman, S., 2008. Identification of the Plastic Material Behaviour through Full-Field Displacement Measurements and Inverse Methods. Dissertation. Vrije Universiteit Brussel.

Cooreman, S., Lecompte, D., Sol, H., Vantomme, J., Debruyne, D., 2007. Elasto-plastic material parameter identification by inverse methods: calculation of the sensitivity matrix. Int. J. Solid Struct. 44, 4329-4341. https://doi.org/10.1016/j.ijsolstr.2006 11.024 .

Endelt, B., Danckert, J., 2009. Identification of friction coefficients and hardening parameters using optimization methods coupled with a 3D finite element code. J. Mater. Process. Technol. 209, 4005-4010. https://doi.org/10.1016/j.jmatprotec.2008.09. 028.

Endelt, B., Nielsen, K.B., 2004. Inverse modeling based on an analytical definition of the Jacobian matrix associated with Hill's 48 yield criterion. In: Stören, S. (Ed.), Proceedings of the 7th Esaform Conference on Material Forming (ESAFORM'04), Trondheim, Norway.

Endelt, B., Nielsen, K.B., Danckert, J., 2005. Analytic differentiation of Barlat's 2D criteria for inverse modelling. In: AIP Conference Proceedings 778. pp. 789-794. https://doi. org/10.1063/1.2011318.

Hill, R., 1948. A theory of the yielding and plastic flow of anisotropic metals. Proc. R. Soc. A. Math. Phys. Eng. Sci. 193, 281-297. https://doi.org/10.1098/rspa.1948.0045.

Marquardt, D.W., 1963. An algorithm for least-squares estimation of nonlinear parameters. J. Soc. Ind. Appl. Math. 11, 431-441. https://doi.org/10.1137/0111030.

Menezes, L.F., Teodosiu, C., 2000. Three-dimensional numerical simulation of the deep drawing process using solid finite elements. J. Mater. Process. Technol. 97, 100-106. https://doi.org/10.1016/S0924-0136(99)00345-3.

Oliveira, M.C., Alves, J.L., Menezes, L.F., 2008. Algorithms and strategies for treatment of large deformation frictional contact in the numerical simulation of deep drawing process. Arch. Comput. Methods Eng. 15 (2), 113-162. https://doi.org/10.1007/ s11831-008-9018-x.

Pereira, A.F.G., Prates, P.A., Sakharova, N.A., Oliveira, M.C., Fernandes, J.V., 2015. On the identification of kinematic hardening with reverse shear test. Eng. Comput. 31, 681-690. https://doi.org/10.1007/s00366-014-0369-7.

Prates, P.A., Oliveira, M.C., Fernandes, J.V., 2014. A new strategy for the simultaneous identification of constitutive laws parameters of metal sheets using a single test. Comput. Mater. Sci. 85, 102-120. https://doi.org/10.1016/j.commatsci.2013.12. 043.

Prates, P.A., Oliveira, M.C., Fernandes, J.V., 2015. On the equivalence between sets of parameters of the yield criterion and the isotropic and kinematic hardening laws. Int. J. Material Form. 8, 505-515. http://doi.org/10.1007/s12289-014-1173-z.

Prates, P.A., Pereira, A.F.G., Sakharova, N.A., Oliveira, M.C., Fernandes, J.V., 2016a. Inverse strategies for identifying the parameters of constitutive laws of metal sheets. Ann. Mater. Sci. Eng. 2016 Article ID 4152963. https://doi.org/10.1155/2016/ 4152963.

Prates, P.A., Oliveira, M.C., Fernandes, J.V., 2016b. Identification of material parameters for thin sheets from single biaxial tensile test using a sequential inverse identification strategy. Int. J. Material Form. 9 (4), 547-571. https://doi.org/10.1007/s12289-0151241-z.

Reis, L.C., Prates, P.A., Oliveira, M.C., Fernandes, J.V., 2017a. Inverse identification of the Swift law parameters using the bulge test. Int. J. Material Form. 10 (4), 493-513. https://doi.org/10.1007/s12289-016-1296-5.

Reis, L.C., Oliveira, M.C., Santos, A.D., Fernandes, J.V., 2017b. On the determination of the work hardening curve using the bulge test. Int. J. Mech. Sci. 105, 158-181. https://doi.org/10.1016/j.ijmecsci.2015.11.009.

Reis, L.C., Prates, P.A., Oliveira, M.C., Santos, A.D., Fernandes, J.V., 2017c. Anisotropy and plastic flow in the circular bulge test. Int. J. Mech. Sci. 128-129, 70-93. https:// doi.org/10.1016/j.ijmecsci.2017.04.007.

Santos, A.D., Teixeira, P., Barata da Rocha, A., Barlat, F., Moon, Y.H., Lee, M.-G., 2010. On the determination of flow stress using bulge test and mechanical measurement. In: Barlat, F., Moon, Y.H., Lee, M.G. (Eds.), 10th Int. Conf. NUMIFORM. American Institute of Physics, Pohang, Republic of Korea, pp. 845-852. https://dx.doi.org/10. $1063 / 1.3457644$.

Swift, H.W., 1952. Plastic instability under plane stress. J. Mech. Phys. Solid. 1, 1-18. https://doi.org/10.1016/0022-5096(52)90002-1.

Teodosiu, C., Bouvier, S., 2001. Selection and identification of elastoplastic models for materials used in the benchmarks. In: 18-months Progress Report, "3DS, Digital Die Design System".

Voce, E., 1948. The relationship between stress and strain for homogeneous deformation. J. Inst. Met. 74, 537-562. 